\title{
Resignificación del pensamiento de Paulo Freire en la praxis pedagógica
}

\author{
Re-signification of Paulo Freire's thought in Pedagogical Praxis \\ Resignificação do pensamento de Paulo Freire sobre a praxe \\ pedagógica
}

Vicente Iván Cruz Jérez

Especialista en Comunicación y Creatividad para la Docencia

Escuela Normal Superior de Neiva

vicvancruxe@gmail.com

\section{Resumen}

El 19 de septiembre de 2021 se conmemoró el primer centenatrio del natalicio de uno de los pedagogos y filósofos de la educación más influyentes del siglo XX. Este acontecimiento desató una serie de eventos académicos y culturales a nivel mundial en los medios de comunicación, organizaciones populares, instituciones educativas y docentes, con el propósito de exaltar la memoria y el trabajo del gran maestro latinoamericano por su significativo aporte al desarrollo de la educación Popular que pretende la liberación de los oprimidos.

Para la Escuela Popular Claretiana (EPC), conocida también como Filodehambre y Programa Formación Complementaria (PFC) de la Escuela Normal Superior de Neiva (ENSN), resultaba imposible no vincularse a la celebración de tan magno evento, porque éstas dos experiencias vienen implementando los principios filosóficos, pedagógicos y metodológicos de Paulo Freire en su praxis educativa, tratando decantar una propuesta pedagógica liberadora con pertinencia rural, urbana marginal y para la inclusión. Esta es la razón principal para socializar los aspectos sobresalientes de la praxis educativa que ponen en evidencia el pensamiento pedagógico libertario freireano, impactando, de este modo en la vida de los niños, niñas y jóvenes de los sectores populares de Neiva y 
departamento del Huila, que acuden a éstas dos escuelas, buscando ser valorados y reconocidos como sujetos sociales.

Palabras clave: Praxis pedagógica, pensamiento freireano, currículo.

\begin{abstract}
September 19, 2021, commemorated the first centenary of the birth of one of the most influential pedagogues and educational philosophers of the twentieth century. This event unleashed a series of academic and cultural events worldwide in the media, popular organizations, educational institutions, and teachers, to exalt the memory and the work of the great Latin American teacher for his significant contribution to the development of Popular education that aims at the liberation of the oppressed.
\end{abstract}

For the Claretian Popular School (EPC), also known as Filodehambre and Complementary Formation Program (PFC) of the Escuela Normal Superior de Neiva (ENSN), it was impossible not to be linked to the celebration of such a great event, because these two experiences have been implementing the philosophical, pedagogical and methodological principles of Paulo Freire in their educational praxis, trying to decant a liberating pedagogical proposal with rural, urban marginal relevance and for inclusion. This is the main reason to socialize the outstanding aspects of the educational praxis that highlight the Freirean libertarian pedagogical thinking, thus impacting the lives of children and young people of the popular sectors of Neiva and Huila department, who attend these two schools, seeking to be valued and recognized as social subjects.

Keywords: Pedagogical praxis, Freirean thought, curriculum.

\title{
Resumo
}

Em 19 de setembro de 2021, foi comemorado o primeiro centenário do nascimento de um dos mais influentes educadores e filósofos do século XX. Este acontecimento desencadeou uma série de eventos acadêmicos e culturais em todo o mundo, na mídia, organizações populares, instituições de ensino e professores, com o objetivo de exaltar a memória e a obra do grande professor latino-americano por sua significativa contribuição para o desenvolvimento da educação que busca a libertação dos oprimidos.

Para a Escola Popular Claretiana (EPC), também conhecida como Filodehambre e o Programa de Formação Complementar (PFC) da Escola Normal Superior de Neiva (ENSN), era impossível não estar vinculado à celebração de um evento tão grande, porque essas dois experiências vêm implementando os princípios filosóficos, pedagógicos e metodológicos de 
Paulo Freire na sua práxis educativa, procurando decantar uma proposta pedagógica libertadora com pertinência rural, urbana marginal e para a inclusão. Este é o principal motivo para socializar os aspectos marcantes da prática educativa que evidenciam o pensamento pedagógico libertário freiriano, impactando assim a vida de crianças e jovens dos setores populares de Neiva e do Estado do Huila, que frequentam essas duas escolas, buscando ser valorizados e reconhecidos como sujeitos sociais.

Palavras-chave: Práxis pedagógica, pensamento freiriano, currículo.

El contexto es la base sobre la cual se estructura el sentido de este ensayo, pues, al describir los datos, los hechos, los fenómenos relacionados con estas dos experiencias educativas, se pretende "definir la naturaleza del escenario y de los antecedentes en el marco de los cuales se han desarrollado" (Serje, 2011), constatando las causas que han posibilitado la permanecia y/o modificaciones del pensamiento freireano en el transcurso de las últimas cuatro décadas, como también las conexiones más allá del aula.

Dicho de otro modo, lo que determina el valor de las dos experiencias es su ubicación, siempre renovada, en el contexto tanto a nivel mundial, como del movimiento político y social que vive América Latina que se agudiza a partir de los años sesenta del siglo pasado, porque allí surge la necesidad de una lectura interpretativa para identificar, las causas estructurales de la crisis económica, politica y cultural que caracteriza esa realidad contextual que, en términos generales alude a los procesos de globalización, industrialización, explotación de los recursos naturales, relaciones obrero patronales injustas y opresivas, escasa o nula participación del pueblo en las políticas del estado, falta de oportunidades para que los sectores populares alcancen condiciones de vida digna.

El análisis de las causas conduce a señalar responsables, de ahí la crítica frontal a la institución escolar por su distanciamiento de la realidad y su aparente neutralidad frente a las políticas del estado, "aunque no logra impactar en sus estructuras" (Puigros, 2005). De este análisis surgen alterantivas, unas tan extremas que propmueven su muerte (Ilich, 1985) y otras, en la óptica de Paulo Freire, quien considera que la educación debe contribuir a la creación de una conciencia emancipatoria en los sujetos de la acción educativa para que asuman la responsabilidad de trabajar por el cambio de la sociedad. Por tanto, compete a los maestros y maestras asumir el liderazgo, para lo cual Freire 
habilita al educador a transgredir las reglas de la educación dominante, para ser participe del cambio social, descubriendo, en cada situación histórica en particular, las tareas que puede realizar. La transgresión, sin embargo, no se hará poniendo el acento en el análisis de los metódos y técnicas, sino en el carácter político de la educación. (Puigros, 2005, pág. 21).

En cierto modo, los problemas específicos de la educación quedan planteado con suficiente claridad, los discursos acerca de la dimensión politica de la educación son elocuentes, mientras tanto, lo que falta, dirá Lola Cendales (1987) es "operacionalizar las alternativas" (Colectivo Escuela Poplar, pág. 5).

En consecuencia, la EPC y el PFC, desde su inicio, realizan lectura de contexto, concebido en su dinámica cambiante, como condición fundamental y código de vida académica y cultural, base sobre la cual se consolida la memoria colectiva en la que el tiempo, el espacio y el lenguaje juegan un papel estructurante (Halbwachs,1968), por tanto se considera el pasado, el presente y el futuro como parte de un mismo tiempo de duración (Contreras, 2018), por la imposibilidad de separar el antes y el ahora de los procesos de reflexión-acciónreflexión mediante los cuales se va consolidando el sentido de la vida en las dos instituciones, es decir el curriculum, cuya dinámica se asemeja a la de un movimiento serpentino $y$ en espiral que posibilita su depuración. En el lenguaje están las claves específicas para la comprensión del significado de educación popular, pedagogía crítica, pedagogía de la liberación, epistemolgías del sur global.

La planeación del trabajo pedagógico, la ejecución de las acciones educativas para la construcción de conocimiento, siempre se realizan en tiempo presente, de modo que es posible recordar el futuro (Contreras, 2018) aunque parezca un contrasentido, es la esencia de la lógica de la contracultura, de la lógica de-colonial, la de las epistemologías del sur global (Sousa, B. 2009) dentro de las cuales, se sitúa el pensamiento pedagógico-político de Paulo Freire.

La lectura actual del conexto permite afirmar que la crisis sanitaria desatada por el coronavirus covid-19 ha puesto en evidencia una crisis mucho mayor y más compleja, la de un sistema económico, político y social basado en la codicia, el lucro y la mercantilización de la vida, el cual desafía y exige a los educadores y educadoras rebeldes, asumir el reto de seguir caminando a pesar del riesgo que significa, no solamente el contagio sino de la violencia con la que el sistema imperante, a través de sus instituciones, trata de 
contrarrestar y aniquilar toda manifestación, todo movimiento social, todo pensamiento alterno que obtaculise sus perversos propósitos. La fortaleza requerida para pervivir en medio del conflicto está contenida en los postulados de la Pedagogía de la esperanza y Pedagogía de la autonomía, que se han constituido en la base referencial que soporta la utopia de la educación como práctica de la libertad, a través de la que se asume

la historia como posibilidad, lo cual significa que mañana no es algo que necesariamente va a suceder, ni una simple repetición del día de hoy con una cara suficientemente maquillada para que pueda continuar siendo lo mismo, pues, no se entiende la esperanza sin un futuro al que hay que hacer, construir y dar forma. (Freire, 1997)

La realidad asumida desde la esperanza, en la perspectiva de la liberación de toda opresión, se presenta, como bien lo expresa Hugo Zemelman, en el ensayo "Subjetividad y sujetos sociales" de Alfonso Torres (2005), como una oportunidad para reivindicarnos en la condición de actores de la nueva historia en la que la subjetividad juega un incuestionable papel en los procesos de construcción de conocimiento, idea que reitera Freire en la introducción a "Los profesores como intelectuales", en la obra de Giroux (1997, pág. 28). Allí plantea:

Que la subjetividad ha de desempeñar un importante papel en el proceso de transformación.

Que la educación se convierte en algo relevante en la medida en que ese papel de la subjetividad se ve como una tarea histórica y política necesaria.

Que la educación pierde significado si no se entiende que, como todas las prácticas, está siendo sometida a limitaciones. Si la educación pudiera hacerlo todo, no habría razón para hablar acerca de sus limitaciones. Si la educación no pudiera hacer nada, tampoco tendría mucho sentido hablar de sus limitaciones.

La historia como posibilidad significa nuestro rechazo a aceptar dogmas, como nuestro rechazo a aceptar la domesticación del tiempo. Hombres y mujeres hacen la historia que es posible, no la historia que a ellos les gustaría hacer, ni la historia que a veces se les dice que debería hacerse. (1997, pág. 30)

Paulo Freire acompaña el caminar de la Escuela Popular Claretiana - Filodehambre

Para entender las circunstancias en las que llegan los primeros 
educadores a ese periférico e inhóspito lugar de la ciudad de Neiva, denominado filo-dehambre, en 1980, es necesario acudir a la descripción y a la hermenéutica moderna para recuperar la esencia de la intencionalidad de esa opción repleta de ética y compromiso; es la época en la que la crisis de las utopías del cristianismocatolicisismo, de las luchas populares por reivindicar los derechos ciudadanos, de la sobrepolitización del movimiento social, del análisis de las estructuras y las coyunturas, está en la cima. En Colombia es la crisis existencial de los sin tierra, de los invisibilizados de la sociedad y el estado, de los perseguidos que se desplazan, por todo el país, huyendo de la violencia, tratando de encontrar un espacio para vivir, aunque sea a la intemperie.

El lenguaje popular de la crisis de la iglesia católica se expresa con frases tales como colgar los hábitos, abandonar las sotanas, curas y monjas ranclados (escapados) de los conventos y otras de mayor elaboración mental y sentido, como opción preferencial por los pobres, trabajo con comunidades eclesiales de base; mientras que, desde posturas civiles y académicas, el lenguaje alude a las preocupaciones sobre la relación entre estructuras sociales y acción colectiva, entre condiciones objetivas de existencia y procesos subjetivos; entre circunstancias y actores sociales, tratando de resolver la tensión entre la objetividad de la racionalidad explicativa del estructuralismo, funcionalismo y marxismo para dar paso a la dimensión subjetiva de los actores sociales (Torres, 2017, pág. 1).

Las maestras y maestros que llegan a Filodehmabre, son personas que, habiendo estado vinculadas, de algún modo, a la vida religiosa por prolongados $\mathrm{o}$ cortos períodos, la abandonan con el fin adelantar un trabajo social de mayor significado dadas las posibilidades que abre tanto, el Concilio Vaticano II y la Teología de la liberación, como también los planteamientos de la educación liberadora de Paulo Freire. De este modo, en filodehambre, confluyen ideales, culturas, experiencias de formación que dan lugar al diálogo de saberes y una convocatoria especial a la consolidación de una comunidad de aprendizaje mediante una praxis educativa que asume la vida como contenido y método para construir conocimiento.

El encuentro con la comunidad barrial se da en un momento de completa incertidumbre, pues está intentando consolidarse como asentamiento subnormal en un lote de propiedad privada. La oferta de los educadores, aunque tenga el tinte asistencialista, es doble: ¿desean un puesto de salud o una escuela? Haciendo uso de su sentido práctico, opta por la escuela. Así comienza el diálogo y 
el compromiso poniendo sobre la meza las condiciones que posibiliten una escuela insertada en la vida de la comunidad barrial con la intención de hacer camino conjuntamente.

El aparente contra-sentido de recordar el futuro, expresado en párrafos anteriores, cobra vigencia a la hora de exponer, en esta reflexión, los fundamentos pedagógicos freireanos, que de manera implícita estuvieron latentes al iniciar la experiencia educativa de Filodehambre. Los verbos en infinitivo de cada postulado, al no definir el tiempo, dan la posibilidad de ser entendidos en el sentido del ayer, del hoy y del porvenir. De los 27 postulados contenidos en Pedagogía de la Autonomía, se mencionan los más significativos:

1. Enseñar exige disponibilidad para el diálogo, 2. Enseñar exige saber escuchar. 3. Enseñar exige compromiso. 4. Enseñar exige comprender que la educación es una forma de intervención en el mundo. 5. Enseñar exige seguridad, competencia profesional y generosidad. 6 . Enseñar exige una toma consciente de decisiones. 7. Enseñar exige reconocer que la educación es ideológica. 8. Enseñar exige querer bien a los educandos. 9. Enseñar no es transferir conocimientos, sino generar las condiciones para producirlos (Freire, 2004).

De aquí en adelante el proyecto educativo va adquiriendo consistencia y coherencia mediante la reflexión en la acción para volver a ella renovados; en este procedimiento, el diálogo con Paulo Freire se va haciendo familiar $y$ se resemantiza su contenido por cuanto los sujetos sociales (niños, niñas, padres y madres de familia que participan de la experiencia de Filodehambre) y el contexto, difieren culturalmente de aquellos en los que Freire adelantó su trabajo pedagógico.

Las frases "Filodehambre nace $y$ crece con la comunidad" y "Escuela Popular Claretiana, una idea que camina" afianzan el sentido de las expresiones expuestas en el preámbulo acerca de la lógica de la espaciotemporlidad, porque cuando se afirma que "los sectores populares descubren su identidad de clase, perciben sus propios intereses y se se organizan para defenderlos como protagonistas de las transformaciones históricas" (Colectivo Escuela Popular, 1987, pag 36) pareciera que el ideal se va concretando y redifiniendo paulatimante, mediante la estrategia de ensayo error, por tanto, no se concibe como meta final, sino como acontecimiento en el aquí y ahora.

La fidelidad renovada y renovadora de los postulados de Freire, en la E.P.C, Filodehambre, se evidencia en las transformaciones, no sólo en el aspecto físico de la escuela y del sector barrial, sino principalmente 
en los actores del proceso mediante una participación real y efectiva, tal como se puede constatar en los testimonios contenidos en el libro La participación en la escuela, entretejiendo pasado, presente $y$ futuro (Colectivo Escuela Popular, 2009) como también en las cuatro estrategias metodológicas del proyecto educativo:

Construcción de un currículo propio a partir de la lectura que hacen los actores de su entorno natural y social ( $\mathrm{La}$ exploración de la realidad, personal, social y natural es el punto de partida para planear, desarrollar, evaluar y socializar los proyectos pedagógicos de aula).

Organización cooperativa de los actores en torno a la construcción de conocimiento, valores y cultura (haciendo educación en una perspectiva crítica, progresista, nos obligamos, por coherencia, a generar, a estimular y a favorecer en la propia práctica educativa, el ejercicio del derecho a la participación por parte de todos los que están directa 0 indirectamente vinculados con el quehacer educativo).

Articulación de la escuela con la comunidad y las organizaciones sociales (El proyecto pedagógico de la escuela y el desarrollo de una propuesta de trabajo comunita- rio y barrial, contribuyó a reivindicar para los pobladores sus derechos fundamentales de tener una vivienda digna con servicios básicos mediante la formación de grupos y organizaciones que se fueron vinculando a un movimiento cívico de carácter regional y nacional) (Colectivo de trabajo barrial, 1992).

Formación permanente de las educadoras y educadores (No podemos separar la práctica de la teoría. La práctica es para Freire la reflexión que deviene acción, y es sólo la acción la que nos puede llevar a la transformación social que la pedagogía liberadora busca". No vamos a la práctica con una teoría para validarla, sino que la teoría, los procedimientos y la reflexión constituyen la postura epistemológica, que se construye en la reflexiónacción reflexión-. Cada uno a su nivel y dependiendo del grado de participación va logrando una comprensión del mundo que le permite superar la captación ingenua de la realidad.) (Colectivo Escuela Popular, 1987, pág. 36)

Para cerrar esta primera reflexión sobre la EPC Filodehambre, vale la pena mencionar algunos alcances:

La lectura crítica de la realidad, ha posibilitado la estructuración de un esquema de pensamiento que lleva en sí mismo la 
construcción de un nuevo sentido de sujeto y de sociedad. "No podemos decir que hicimos invasiones, porque somos colombianos y estamos en nuestro propio territorio. Si algo nos ha enseñado la escuela, es el verdadero sentido de patria." (Alirio Ramírez. Padre de familia).

Este pensamiento ha orientado la redefinición del papel de la escuela contribuyendo a resignificar, tanto, el concepto de pedagogía, como la construcción de sentido y la búsqueda de la libertad.

Nuestra escuela es diferente en su metodología, que no quiere que los niños aprendan a repetir lo que está en los libros. $\mathrm{Al}$ niño se le va enseñando que puede producir conocimiento, que tiene capacidad de expresarse sobre lo que está sintiendo y que puede mejorar y cambiar su forma de ser en la escuela y en el hogar" (Graciela Méndez - madre de familia)

Al compartir los proyectos de vida, tanto de los educadores como de los educandos y demás participantes de la experiencia de Filodehambre, se ha ido tejiendo el sentido de lo colectivo, la autoconciencia y la conciencia social, orientando las metas personales y grupales hacia la conquista progresiva de la autoestima para posicionarse como actores sociales, en una perspectiva liberadora.
El aprendizaje en la escuela me ayudó a abrirme a otros espacios tales como mi trabajo en los hogares comunitarios, en mi barrio como miembro en la junta de acción comunal; aprendí a sentirme libre, autónoma y decidida, pues supe que estar allí no es solo aportar sino formarme como persona, mujer, ciudadana" (Yaqueline Piedrahita- madre de familia)

\section{Limitaciones e inacabamientos}

Una experiencia de este tipo dentro de la educación formal se ve limitada en sus posibilidades por las políticas del estado neoliberal, restándole autonomía para decidir por si misma en todos y cada uno de los aspectos del proyecto educativo.

La mayoría de maestros asignados por el estado no están en la capacidad para atender las urgencias que la vida cotidiana presenta en un sector donde confluyen todo tipo de violencias, desigualdades y carencias.

Paulo Freire en el programa de formación complementaria de la Escuela Normal Superior de Neiva

El movimiento pedagógico que inicia en Colombia a finales de 1970 y la promulgación la Ley General de Educación o ley 115 de 1994, constiuyen el marco en el que se da el encuentro de la Escuela Popualr Claretiana con la Escuela Normal Superior de Neiva, 
por cuanto ambas instituciones educativas, tienen entre sus propósitos ampliar las relaciones con otros espacios académicos y sociales para ir consolidando "una plataforma desde la cual sea posible reinventar nuevas maneras de lucha contra el capitalismo neoliberal" (Mclaren, 1997).

En el año 2001, se promulga la ley 715 , mediante la cual se fucionan instituciones educativas y la EPC opta por integrarse a la ENSN, como una de las sedes, sin renunciar al compromiso y fidelidad al ideario políticopedagógico que sustenta su proyecto educativo, de modo que el encuentro se fortalece, porque tanto la una y la otra se complementan en la praxis educativa.

La opción de la EPC como la Escuela Normal Superior de Neiva, por la pedagogía crítica, no es coyuntural, está ligada a todo un movimiento histórico, estructural y sostenido que se remonta más allá de la teoría crítica de la sociedad, que nace en la escuela de Frankfurt.

En este recorrido debe entenderse la relación con el pensamiento emancipatorio de Simón Rodríguez, en lo que respecta a la formación de ciudadanía, la asunción de responsabilidades para hacerla posible y la lucha por los derechos para la dignificación de la vida; con Celestín Freinet, quien plantea la vida como contenido y método de aprendizaje $y$ el trabajo cooperativo que suscita los valores de la solidaridad y reconocimiento de la diversidad de la que estamos hechos. Con Paulo Freire, por el papel dialogante del maestro con las y los estudiantes para concertar y promover procesos de formación en la autonomía y la participación como valores esenciales en la construcción de currículos que abordan problemas vitales, contextualizados, que contribuyen con procesos de transformación personal y social, es decir con la dimensión politica de la práctica pedagógica.

Concretar el discurso de las pedagogías críticas en el PFC, ha sido posible mediante la implemntación de los Proyectos Pedagógicos de Aula, concebidos como procesos de investigación que se planean, desarrollan, sistematizan, evalúan y socializan, mediados por la organización cooperativa cuyo valor sustancial es la participación. Estos proyectos se estructuran en tres etapas:

1. Concertación. Esta pretende hacer efectivos los principios básicos para democratizar la escuela y el conocimiento, en tal sentido, el sujeto que participa de los procesos de aprendizaje ocupa un lugar preferencial, lo cual exige del maestro la responsabilidad de respetar, reconocer y valorar los saberes con los que llegan los estudiantes, sobre todo los de las 
clases populares, saberes construidos en la práctica sociocultural de la vida cotidiana (Freire, 2006). Estos se expresan, en forma oral, gráfica o escrita, dependiendo de las capacidades de cada uno; la comunicación de los saberes individuales, se sintetizan en un texto colectivo, que al analizarlo genera discusiones y cuestionamientos que dan origen a preguntas de interés, a las que deben supeditarse los propósitos del aprendizaje, pues

se trata de que tanto los métodos como los contenidos y las formas de organización escolar se correspondan realmente con esos intereses y necesidades esenciales de los estudiantes en función de las necesidades de la sociedad a la que pertenecen. (Freinet, 1977)

Una vez priorizadas las preguntas y seleccionada la de mayor interés, se procede a elaborar un plan de investigación con propósitos bien definidos, sustentados en las nuerociencias, que con sus aportes pemtiten que los procesos de aprendizaje se correspondan con las lógicas con las que aprenden niños, niñas y jóvenes; del mismo modo se prevén las fuentes y se especifican los instrumentos para recolectar la información, las formas de sistematización como también los productos para la socialización.

Una vez que se ha logrado concretar el plan de investigación, deviene la organización cooperativa para asumir, de manera consciente, las responsabilidades que exige el desarrollo del plan. En consecuencia, se elaboran los acuerdos colectivos en los que se sintetizan las responsabilidades y los derechos de cada uno de los participantes, que indudablemente contribuyen a la consolidación de los valores de cooperación, solidaridad, responsabilidad, autonomía, autoestima, respeto y reconocimiento de la diversidad.

La sistematización de este proceso se registra en la matriz epistemológica y las relatorías diarias que son leídas al inicio de cada sesión, de tal manera que las y los estudiantes, maestras y maestros se apropien de su contenido a la vez que sirven de referente nemotécnico para tener presente el proceso metodológico y el contenido.

En esta etapa, la participación es progresiva hasta llegar a ser consciente, en la medida que el proceso de estructuración del proyecto pedagógico avanza. Comienza, así, ha manifestarse el sentido de la autonomía y de la autoestima, que son las bases de la formación personal y ciudadana. De este modo cobra sentido el postulado "educar no es transmitir conocimientos, sino generar las condiciones para producirlos" (Freire, 2006) y más aún, entender que el acto de aprender no es mecánico, construye conciencia, estimula la 
cooperación, la creatividad y la imaginación.

2. Desarrollo: Aproximarse a la realidad social o natural haciendo preguntas, es asumir la lógica con la que aprenden los niños, niñas y jóvenes, es decir que

el inicio del desarrollo del pensamiento lógico precede a la "edad de la razón" e incluso al lenguaje y que hay continuidad entre el niño que construye su mundo y el científico que construye una teoría acerca de ese mundo. (Piaget, 1971, pág. 165)

En este sentido, las experiencias de aprendizaje las asumen maestros y estudiantes desde la propia corporalidad (subjetividades), con ella entran en contacto con el mundo exterior para observarlo, describirlo, cuantificarlo, compararlo, representarlo, hacer inferencias, elaborar nociones, concepciones y establecer relaciones comunicativas mediante múltiples lenguajes a fin de participar en la construcción social de la realidad.

Cada experiencia planeada permite recoger la información requerida, encaminada a encontrar respuestas a los interrogantes que motivan la investigación educativa. En esta dinámica adquieren un profundo sentido las historias de vida, las autobiografías, los recorridos por la localidad barrial o la vereda; realizar entrevistas, hacer visitas a las familias, aplicar encuestas, iniciar procesos de cartografía social (levantar mapas, ubicar allí los sitios más importantes), hacer experimentos, registrar datos con instrumentos técnicos. Es una forma esencial de leer la realidad natural y social en la que se habita para construir conocimiento $y$ apropiación del territorio.

El salón de clase se convierte en el espacio para la sistematización mediante la que se organiza la información, se analiza y se complementa con los aportes del conocimiento constituido (contenido en las asignaturas del plan de estudios), se hacen síntesis, se elaboran documentos de acuerdo con las capacidades y niveles de comprensión de las y los estudiantes. En los informes, cartillas, folletos, dibujos, cuentos, álbumes, se puede verificar los aprendizajes como yambien las formas de recreación, reinvención, apropiación y afianzamiento de los conocimientos.

Estos documentos reflejan la capacidad y la creatividad de los participantes para comunicar los aprendizajes. Los niños y niñas de los primeros años de escolaridad inician el proceso de escritura a partir de las experiencias vividas y de la necesidad de usar otro tipo de lenguaje para la comunicación (código escrito). De este modo, el aprendizaje no está en función de una nota o calificación con el propósito de ser promovidos de un nivel a otro, sino en la 
perspectiva de la formación de la persona y del ciudadano- como sujeto social.

3. Socialización. En esta etapa se evidencia cómo y hasta qué punto se han dado esos circuitos de articulación sucesiva, entre el sujeto individual que participa en un proceso de construcción colectiva y el contexto natural y socio-cultural, a los que se refiere Ardila (2005) a propósito de la construcción de territorialidad, puesto que en la socialización, las y los estudiantes comparten los aprendizajes alcanzados, no sólo los referidos a la dimensión académica-cognitiva, sino a todos cuantos intervienen en esa relación compleja entre la vida de los sujetos (las subjetividades y objetividades) y la organización escolar. Son ellos y ellas quienes, a través del arte, en sus múltiples formas, comunican los alcances, las limitaciones y los efectos en sus propias vidas y el contexto.

Desde esta mirada es posible entender la puesta en escena en la que se destacan el teatro, la danza, la literatura, la música, la pintura, el dibujo y la producción escrita que contribuyen a la construcción social de la realidad, es esa otra forma de poder (ser, sentir, pensar y actuar).

El debate que se genera después de la puesta en escena estimula los aportes de la comunidad (padres de familia, vecinos de la comunidad barrial, veredal, autoridades educativas y civiles), con sus cuestionamientos y críticas; por una parte, redireccionan, replantean y enriquecen los conocimientos construidos y, por otra evidencian la relatividad de los mismos y el inacabamiento de los sujetos que aprenden.

En suma, la socialización se constituye en un foro en el que aflora la dimensión política, histórica, pedagógica y cultural de la educación popular como también de la educación formal.

\section{Epílogo}

Parafraseando a Margarita Serjé (2011), podría decirse que el nuevo escenario global, en el que es posible leer la crisis del capilalismo depredador de la naturaleza y explotador de la fuerza laboral humana, gracias al impacto del coronavirus covid-19, permite elaborar nuevas conceptualizaciones acerca del contexto, por cuanto de la pandemia emerge una nueva categoría, la sindemia, para explicar la relación de acoplamiento de lo social con lo biológico, de modo que queda en evidencia que la crisis sanitaria va ligada a los modos de producción, a las lógicas de la economia global, a la cultura del consumo individualista, a los usos de las nuevas tecnologías de la comuniación y producción, como tambien a las políticas neoconservadoras que se fortalecen con nuevas formas de control del movimiento social alterno. 
Bosquejado asi en nuevo panorama mundial, es posible entender que las polítcas de la OCDE, con respecto a la educación, se perfilan en el marco de la lógica del capitalismo global homogeneizador, que trata de disgregar el tejido social para poder identificar con precisión toda acción contraria o incompatible con sus propósitos y concpetos de desarrollo y progreso, de ahí que la pedagogía crítica sea vista, por el sistema imeprante, como subversiva. Frente a la complejidad del contexto actual, es más que normal que las incertidumbres se agudicen, las preguntas se multipliquen y sea necesario avivar la memoria para pensar que el tiempo transcurrido desde Simón Rodríguez a Paulo Freire, no ha sido en vano. La praxis educativa iluminada por la pedagogía crítica, caracterizada por épocas de efervescencia y opacamiento, se ha fortalecido $y$ su reflejo está en el surgimiento de nuevas epistemologías que dignifican la vida del sur global, que estimulan la generación de experiencias alternativas en la educación formal, educación popular, organismos sindicales, agrupaciones de mujeres, grupos insurgentes, en fin, de posturas radicales e insurgentes.

Desde las dos experiencias pedagógicas expuestas en este artículo, se puede afirmar que Paulo Freire se ha constituido en referente fundamental, que ha hecho posible mantener la esperanza de un nuevo orden social que inicia en una praxis pedagógica al interior del aula, mediante la cual se hace efectivo el reconocimiento y valoración de las $y$ los estudiantes de los sectores populares, que buscan, como necesidad prioritaria, afecto, condición esencial para dar paso a la producción de conocimiento. Pero, más allá de las metodologias al interior del aula, Freire incita, y así ha asumido el equpo de maestras y maestros, a construir un arco de sueño social y de entrada a la esperanza, como bien lo expresa Peter Mclaren en su libro titulado del mismo modo y, a su vez manifiesta la necesidad de construir una plataforma de lucha contra el capitalismo global. Entonces, hay razones suficientes para continuar ampliando y fortaleciendo las relaciones con la Universidad Surcolombiana, DePaul University de Chicago-Usa; Red Estrado, Universidad Pedagógica Nacional, instituciones académicas alternativas; socializaciones periódicas de los resultados de sus investigaciones educativas, como también los foros Escuela de vida y debida. La vida y obra de maestras y maestros que ofrendaron sus vidas en esta lucha por la causa liberadora, están en la memoria y como parte de la historia de estas dos experiencias signficativas.

\section{Referencias}

Ardila, G. (2005). Ingeniería y territorio: Una relación política indisoluble. 
https://revistas.unal.edu.co/ind ex.php/palimpsestvs/article/vie $\mathrm{w} / 8059$

Castro Gómez, S. (2010.) La hybris del punto cero. Bogotá

Colectivo Escuela Popular Claretiana. (1987). FILODEHAMBRE, una experiencia popular de innovación educativa. Bogotá: Presencia Ltda.

Colectivo Escuela Popular Claretiana. (2009). La participación en la escuela, Entretejiendo pasado, presente y futuro. Bogotá: Dimensión Educativa.

Colectivo de Trabajo Barrial. (1992). ORGANIZACIONES BARRIALES Y EDUCACIÓN POPULAR, experiencias organizativas en el sector suroriental de Neiva. Bogotá: Dimensión Educativa.

Contreras, A. (2018). Recordar el futuro, planificación de la comunicación desde la comunicación". Neiva: Universidad Surcolombiana.

Freinet, C. (1977). Por una Escuela del pueblo. Caracas: laboratorio Educativo.
Freire, P. (2010). Pedagogía de la indignación. Madrid

Freire, P. (2006). El grito manso. Buenos Aires

Freire, P. (2004). Pedagogía de la autonomía". Sao Paulo: Paz e Terra SA.

Giroux, H. (1997). Los profesores como intelectuales, hacia la pedagogía crítica del aprendizaje". Buenos Aires: Paidos.

Ilich, I. (1985). La sociedad desescolarizada. México.

Mclaren, P. (1997). Pedagogía crítica y cultura depredadora. México.

Puigros, A. (2005). De Simón Rodríguez a Paulo Freire. Bogotá: Convenio Andrés Bello.

Piaget, J. (1971). Seis estudios de psicologia. Barcelona: Seix Barral

Serjé, M. (2011). El revés de la nación: territorios salvajes, fronteras y tierras de nadie., Bogotá; Uniandes.

Torres, A. (2005). Subjetividad y sujetos sociales en la obra de Hugo Zemelman. Bogotá: U. Pedagógica. 